Pesq. Vet. Bras. 35(12):1002-1008, dezembro 2015

DOI: $10.1590 / \mathrm{S} 0100-736 \mathrm{X} 2015001200010$

\title{
Avaliação da cavidade orofaríngea da tartaruga da Amazônia, Podocnemis expansa (Schweigger, 1812) ${ }^{1}$
}

\begin{abstract}
Thaís B. Silveira², Erica S. Agostinho ${ }^{2}$, Francisco G.A. Santos ${ }^{2}$, Alex C.P. de Oliveira ${ }^{2}$, Luciana S. Medeiros ${ }^{2}$, Yuri K. de Carvalho ${ }^{2 *}$ e Maria A. Miglino ${ }^{3}$

ABSTRACT.- Silveira T.B., Agostinho E.S., Santos F.G.A., Oliveira A.C.P., Medeiros L.S., Carvalho Y.K. \& Miglino M.A. 2015. [Evaluation of the oropharyngeal cavity of Giant South American turtle Podocnemis expansa (Schweigger, 1812).] Avaliação da cavidade orofaríngea da tartaruga da Amazônia Podocnemis expansa (Schweigger, 1812). Pesquisa Veterinária Brasileira 35(12):1002-1008. Centro de Ciências Biológicas e da Natureza, Medicina Veterinária, Universidade Federal do Acre, Campus Universitário, BR-364 Km 4, Distrito Industrial, Rio Branco, AC 69920-900, Brazil. E-mail: ykaracas@yahoo.com.br

Turtles are important as food and economic resources for the Amazon communities and there is a large demand on its meat and eggs. Podocnemis expansa (Giant South American turtle) is the largest freshwater chelonian of South America. This turtle is a widely distributed specie, however its anatomical features are poorly described. The objective of this study was to describe the oropharyngeal cavity of the turtle. Twelve heads of 3 to 8-year-old female and male $P$. expansa turtles with an average body weight of $7.5 \mathrm{~kg}$ were used. $P$. expansa has a sharp and well developed rhamphotheca, which together with a powerful jaw muscles and a large tongue uniformly distributed on the oropharynx floor works for the apprehension and swallowing of food, what ensures good adaptation in different environments.
\end{abstract}

INDEX TERMS: Podocnemis expansa, oral cavity, Giant South American turtle.

RESUMO.- Os quelônios são importantes como fonte alimentar e econômica para as comunidades da região amazônica. Sendo assim, a caça, a pesca e a procura por ovos destes animais tem ocorrido frequentemente. Podocnemis expansa (Tartaruga da Amazônia) é o maior quelônio de água doce da América do Sul. É uma espécie largamente distribuída, porém, nota-se que aspectos anatômicos da espécie são insuficientemente descritos. 0 objetivo desse estudo foi descrever a cavidade orofaríngea desses quelônios. Foram utilizadas 12 cabeças de $P$. expansa adultas, de ambos os sexos, com idade entre 3 a 8 anos, com peso corpóreo de 5 a $22 \mathrm{~kg}$ (média de $7,5 \mathrm{~kg}$ ). P. expansa conta com a presença de rafontecas afiadas e bem desenvolvidas que, associadas à musculatura potente da mandíbula e à língua

\footnotetext{
${ }^{1}$ Recebido em 7 de janeiro de 2015.

Aceito para publicação em 26 de novembro de 2015.

${ }^{2}$ Centro de Ciências Biológicas e da Natureza, Medicina Veterinária, Universidade Federal do Acre (UFAC), Rio Branco, AC 69920-900, Brasil. *Autor para correspondência: ykaracas@yahoo.com.br

${ }^{3}$ Departamento de Cirurgia, Faculdade de Medicina Veterinária e Zootecnia, Universidade de São Paulo (USP), Av. Prof. Dr. Orlando Marques de Paiva 87, Cidade Universitária, São Paulo, SP 05508-270, Brasil.
}

volumosa e bem distribuída no assoalho da orofaringe, atuam na apreensão e deglutição do alimento, garantindo uma maior adaptação em diversos ambientes.

TERMOS DE INDEXAÇÃO: Podocnemis expansa, cavidade orofaríngea, tartaruga da Amazônia.

\section{INTRODUÇÃO}

Os quelônios da Amazônia são importantes para as comunidades da região amazônica, pois funcionam como recurso alimentar - carne, vísceras e ovos - e fabricação de adornos utilizando seus cascos (Moura \& Rodrigues 2007). 0 estudo morfológico de animais silvestres é sempre uma fonte crescente de informações, especialmente quando se trata de espécies que apresentem potencial zootécnico ou valor biológico. Devido a isso, a caça, pesca e busca pelos ovos destes animais tem ocorrido há muitas gerações (Mader 1996, Luz \& Reis 1999, Almeida, 2011). As Portarias $142-\mathrm{N}$ de 30 de dezembro de 1992 e 070 de 23 de agosto de 1996 normatizam a criação comercial de quelônios da Amazônia, a criação em cativeiro, a comercialização de produtos e subprodutos (Brasil 1992, Brasil 1996, Almeida 2011). 
Podocnemis expansa, conhecida como tartaruga da Amazônia, caracteriza-se como o maior quelônio de água doce da América do Sul podendo atingir $80 \mathrm{~cm}$ de comprimento e $60 \mathrm{~cm}$ de largura de carapaça e massa $60 \mathrm{~kg}$ (Melo et al. 2003, Cubas et al. 2014). É uma espécie largamente distribuída, ocorrendo em rios da Colômbia, Venezuela, Guiana, Equador, Peru e Bolívia, já no Brasil encontra-se nas bacias do Amazonas, Araguaia, Tocantins e Branco (Iverson 1992, Almeida 2011). Sua cabeça é pequena e achatada, em animais jovens a possui coloração marrom acinzentada ocorrendo a presença de manchas amarelas em ambos os lados, em fêmeas essas manchas desaparecem e nos machos elas desbotam e escurecem com a idade, já a mandíbula possui coloração marrom claro e o queixo coloração amarelada (Pritchard \& Trebbau 1984, Ernst \& Barbour 1989).

Ernst \& Barbour (1989) e Luz (2005) afirmam quanto a dieta da tartaruga da Amazônia que esta pode se alimentar de frutos, flores, sementes, talos, raízes e folhas arbóreas e herbáceas, além de insetos, crustáceos, moluscos e peixes, tornando-a um animal onívoro oportunista, sendo predominantemente herbívoro. De acordo com Almeida (2011), a apreensão do alimento é feita através do abocanhamento, podendo ocorrer algumas vezes a sucção da presa inteira.

São poucos os trabalhos realizados sobre a descrição das porções anatômicas da tartaruga da Amazônia, inclusive do seu sistema digestório. Diante disso, o presente estudo teve como objetivo descrever os vários aspectos da cavidade orofaríngea de $P$. expansa, buscando informações na literatura que viabilizem uma comparação com outros quelônios e que justifiquem seus hábitos alimentares.

\section{MATERIAL E MÉTODOS}

Este trabalho foi realizado no Laboratório de Anatomia Animal (Centro de Ciências Biológicas e da Natureza) da Universidade Federal do Acre. Para o presente estudo foram utilizadas 12 (doze) cabeças de Tartarugas da Amazônia (Podocnemis expansa) adultas, de ambos os sexos, com idade entre 3 a 8 anos, com a massa corpórea média de 5 a $22 \mathrm{~kg}$ (média de 7,5kg). Os animais foram abatidos em frigorífico sob a licença IBAMA n²15/2011. As cabeças após o abate foram desarticuladas da carcaça através da articulação atlantocciptal, lavadas e acondicionadas em caixa térmica.

Realizou-se então, afim da preservação das peças anatômicas, o processo de formolização das mesmas, em solução de formaldeído a 10\%. Para a avaliação descritiva e quantitativa foi necessário a abertura da cavidade orofaríngea de Podocnemis expansa através da desarticulação tempuro-mandibular, a fim de identificar, mensurar e fotodocumentar as estruturas. A nomenclatura utilizada para denominação das estruturas identificadas teve como base The International Committee on Veterinary Gross Anatomical Nomenclature (2012).

Para a avaliação da morfologia dos tecidos, segmentos de diferentes partes da cavidade orofaríngea como: abertura das coanas e lingua (ápice, porção lateral e porção média) foram retirados e pós-fixados em álcool 70\%. Posteriormente as amostras foram desidratadas em uma série de etanóis em concentrações crescentes (70-100\%), diafanizado em xilol para em seguida serem incluídas em parafina. Após a montagem, as amostras foram cortadas em micrótomo com espessura de $5 \mu \mathrm{m}$ e em seguida corados por Hematoxilina Eosina (HE). Para registro, a fotomicrografia foi realizada por meio do fotomicroscópio Leica DM 2000 e para nomenclatura, fez-se referência conforme International Comitee on Ve- terinary Gross Histological and Anatomical Nomenclature (2005).

Para a realização da microscopia eletrônica de varredura, coletou-se fragmentos de $1,0 \mathrm{~cm}$ de comprimento de diferentes estruturas da cavidade orofaríngea, as quais foram lavadas em água destilada por 20 minutos e fixadas em solução de tetróxido de Ósmio a 1\% (Polyscience ${ }^{\circledR}$ Inc., USA) durante 2 horas. Em seguida, as amostras foram desidratadas em séries crescentes de etanol (50\% a 100\%), durante 30 minutos. A desidratação ao ponto crítico do $\mathrm{CO}_{2}$ foi realizada no aparelho Balzers CPD 020 (FMVZUSP). Utilizou-se bases metálicas de alumínio (stub) para a alocação das amostras, fazendo uso de cola de carbono, sendo assim metalizadas com ouro no aparelho EMITECH K550 (FMVZ-USP) para o registro fotográfico em microscópio eletrônico de varredura (Morgagni 268D - Phillips ${ }^{\circledR}$ ) do Programa de Pós-Graduação da Faculdade de Medicina Veterinária e Zootecnia da Universidade de São Paulo.

\section{RESULTADOS}

A primeira porção da cavidade orofaríngea de Podocnemis expansa é delimitada rostralmente e lateralmente pelo bico queratinizado e caudalmente pela glote, na área da faringe (Fig.1). No Quadro 1 estão descritas as mensurações referentes ao comprimento e largura das narinas superiores, o

Quadro 1. Mensurações em milímetro das cabeças de Podocnemis expansa, em vista dorsal e ventral, adquiridas após abate pelo interesse em sua carne conforme a licença. Média \pm Desvio Padrão, Máximo e Mínimo

\begin{tabular}{|c|c|c|}
\hline & Estruturas & Dimensões (mm) \\
\hline Teto da & Narinas superiores: comprimento & $12,83 \pm 2,21 ; 15,04$ e 10,62 \\
\hline Cavidade & Narinas superiores: largura & $11,58 \pm 1,44 ; 13,02$ e 10,14 \\
\hline \multirow[t]{2}{*}{ Orofaringea } & Extensão da cavidade & $53,83 \pm 4,53 ; 58,36$ e 49,30 \\
\hline & $\begin{array}{l}\text { Estrutura na parte dorsal } \\
\text { (coana + ranfoteca) }\end{array}$ & $23,83 \pm 2,52 ; 26,35$ e 21,31 \\
\hline Assoalho da & Extensão da cavidade orofaríngea & $68,75 \pm 5,51 ; 74,26$ e 63,24 \\
\hline Cavidade & Comprimento da língua & $53,17 \pm 5,64 ; 58,81$ e 47,53 \\
\hline \multirow[t]{3}{*}{ Orofaríngea } & Glote & $11 \pm 0,95 ; 11,95$ e 10,05 \\
\hline & Distância bico/glote & $29,25 \pm 2,45 ; 31,70$ e 26,80 \\
\hline & Abertura da mandíbula & $32,42 \pm 3,15 ; 35,57$ e 29,27 \\
\hline
\end{tabular}

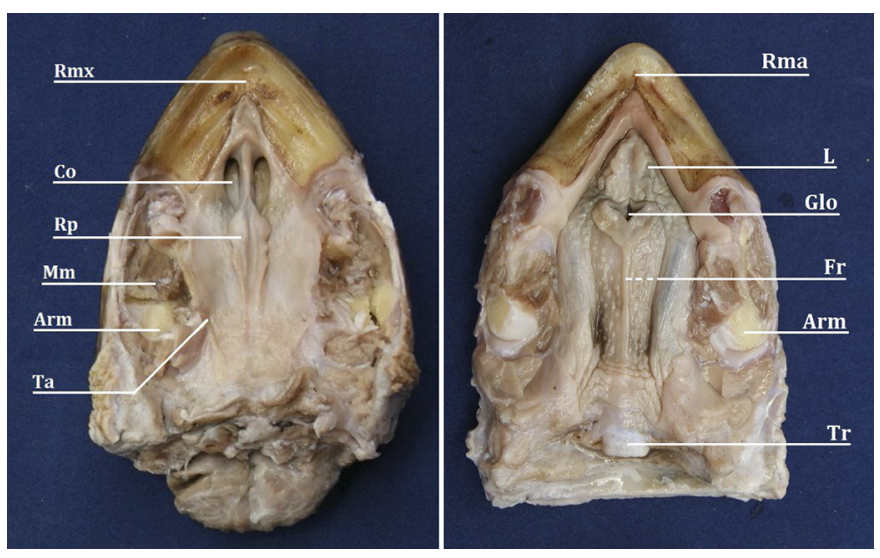

Fig.1. Fotomacrografia das vistas dorsal e ventral da cavidade orofaríngea da Tartaruga da Amazônia (Podocnemis expansa). Barra: $2 \mathrm{~cm} . M m$ = músculo masseter (seccionado horizontalmente), $\mathrm{Co}=$ coanas, $\mathrm{Ta}=$ Tubas auditivas, $\mathrm{Mm}=$ músculo adutor da mandíbula (seccionado horizontalmente), Arm = articulação da mandíbula, $\mathrm{Rm}=$ ranfoteca maxilar, $\mathrm{Rp}=$ rafe palatina, $\mathrm{Rma}=$ ranfoteca maxilar, $\mathrm{L}=$ língua, $\mathrm{Fr}=$ frênulo da língua, $\mathrm{Glo}=$ glote, $\mathrm{Tr}=$ traquéia, $\mathrm{Fa}=$ faringe, $\mathrm{Arm}=$ articulação da mandíbula. Barra: $2 \mathrm{~cm}$. 


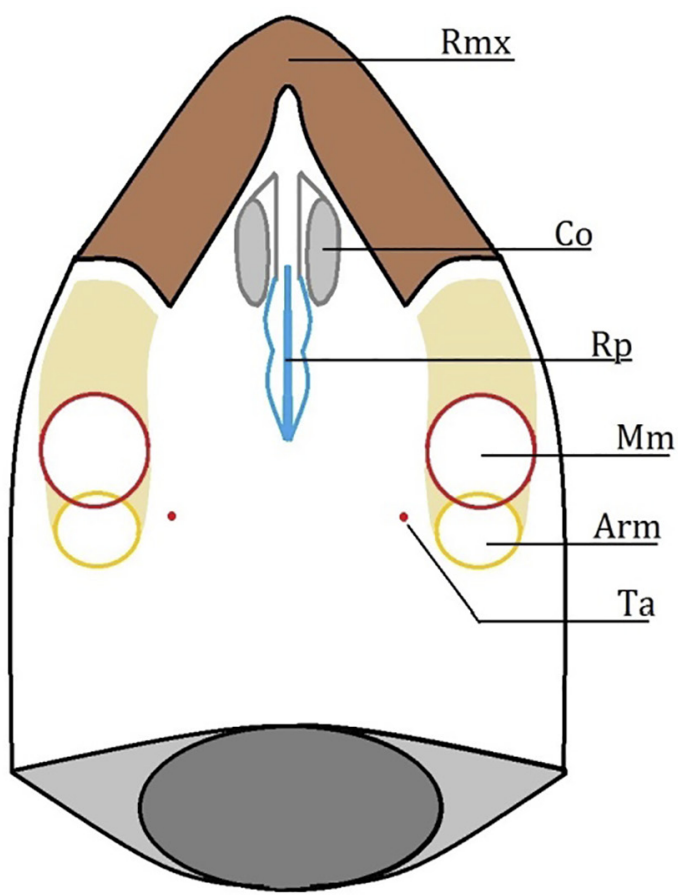

Fig.2. Esquema da vista dorsal da cavidade orofaríngea da Tartaruga da Amazônia (Podocnemis expansa). $M m$ = músculo masseter (seccionado horizontalmente), $\mathrm{Co}=$ coanas, $\mathrm{Ta}=$ Tubas auditivas, $\mathrm{Mm}$ = músculo adutor da mandíbula (seccionado horizontalmente), Arm = articulação da mandíbula, $\mathrm{Rm}=$ ranfoteca maxilar, $\mathrm{Rp}=$ rafe palatina. Barra: $2 \mathrm{~cm}$.

comprimento da extensão do teto, o comprimento referente à união da coana e ranfoteca, o comprimento da língua, da glote, a distância do bico a glote e a abertura da mandíbula.

Verificou-se na vista dorsal da cavidade orofaríngea (Fig.2) a presença de ranfoteca maxilar (Rmx) bem desenvolvida, a qual possui coloração mais escura e que dá continuidade às projeções largas e achatadas, as quais são identificáveis como sendo a rafe palatina (Rp) que se estende até o terço médio da cavidade orofaríngea. Entre tais estruturas é possível observar duas aberturas, reconhecidas como sendo as coanas (Co).

Ao observar microscopicamente a porção dorsal da cavidade orofaríngea, observa-se que a mesma é formada por um epitélio de revestimento pavimentoso estratificado não queratinizado, sendo preenchida superficialmente por tecido conjuntivo frouxo e mais profundamente por tecido conjuntivo denso não modelado. Essa descrição se estende para a membrana que divide a abertura das coanas (Fig.3). Há ainda a presença de grandes quantidades de vasos (sanguíneos e linfáticos) em ectasia vascular.

A mucosa do teto da orofaringe não está distribuída uniformemente, já que não se observa a presença da mesma na porção rostral, onde encontra-se a ranfoteca maxilar. A abertura do par de tubas auditivas $(\mathrm{Ta}$ ) podem ser visualizadas entre o terço médio e o terço caudal do teto, sendo que tais orifícios são projetados na região lateral de cada antímero.

0 músculo adutor da mandíbula $(\mathrm{Mm})$ apresenta-se bilateralmente no terço médio da orofaringe entre o teto e o

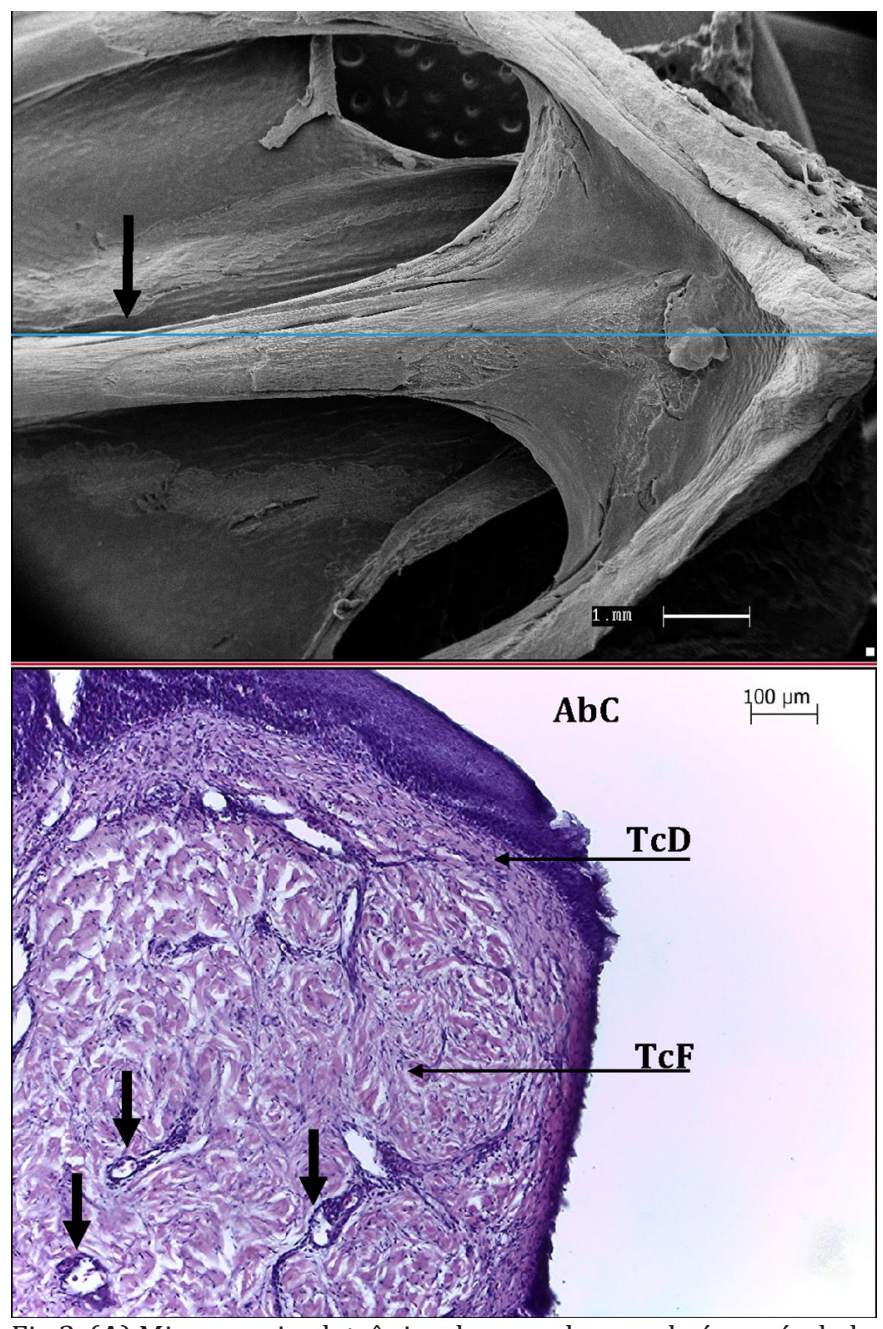

Fig.3. (A) Microscopia eletrônica de varredura onde é possível observar a membrana que divide a abertura das coanas (seta). (B) Fotomicrografia de corte transversal da membrana que divide a abertura das coanas, evidenciando epitélio de revestimento estratificado pavimentoso não queratinizado e grande presença de vasos (setas). Hematoxilina e Eosina. $\mathrm{AbC}=$ abertura das coanas; $\mathrm{TcD}=$ tecido conjuntivo denso; $\mathrm{TcF}=$ tecido conjuntivo frouxo.

assoalho, o qual se divide em duas porções proeminentes, tendo este sido seccionado quando realizada a separação da cabeça e da maxila.

Na vista ventral da cavidade orofaríngea (Fig.4) a mucosa está presente parcialmente, sendo ausente na extremidade rostral do assoalho o que corresponde a ranfoteca mandibular (Rma). A ranfoteca mandibular apresenta uma concavidade a qual se encaixa à ranfoteca maxilar, permitindo uma perfeita oclusão.

A língua (L) é uma estrutura muscular volumosa que ocupa boa parte da cavidade orofaríngea, esta apresenta ainda duas tonalidades de cor: seu ápice tem formato rombo e cor mais escura, já no corpo e raiz a coloração é mais clara. Nela observamos o frênulo da língua (Fr) bastante proeminente, o qual apresenta-se bilateralmente e estende-se ao ápice da língua. É possível identificar uma grande quantidade de papilas, de tamanhos variados e espalhadas por toda a estrutura. 


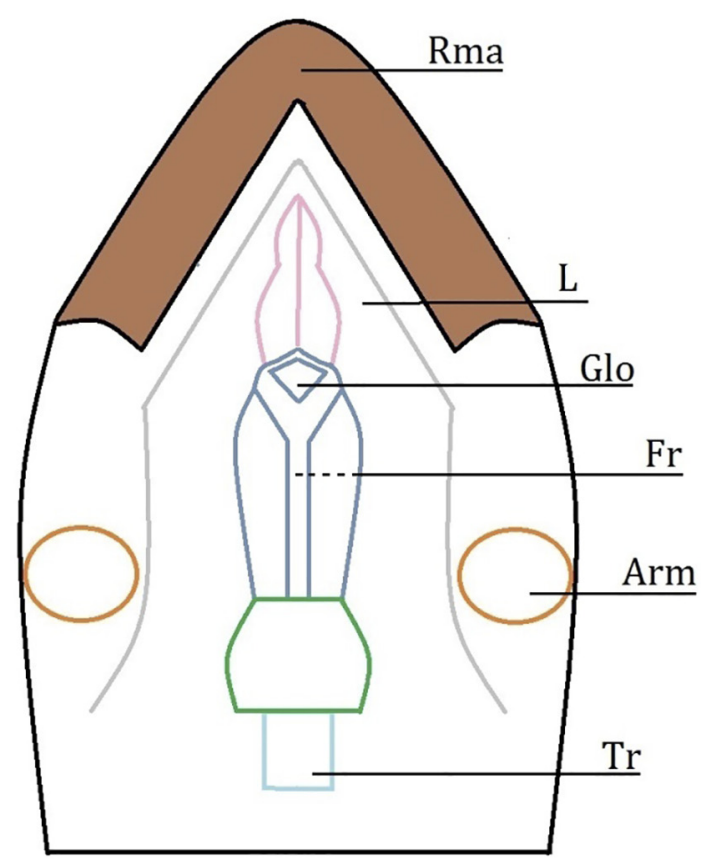

Fig.4. Esquema da vista ventral da cavidade orofaríngea da Tartaruga da Amazônia (Podocnemis expansa). $\mathrm{Rma}=$ ranfoteca maxilar, $\mathrm{L}=$ língua, $\mathrm{Fr}=$ frênulo da língua, Glo = glote, $\mathrm{Tr}=$ traquéia, $\mathrm{Fa}=$ faringe, Arm = articulação da mandíbula. Barra: $2 \mathrm{~cm}$.

Imagens da microscopia da língua em corte transversal permitiram a identificação dessas projeções no epitélio, as quais são reconhecidas como papilas linguais de formato abaulado e que possuem características mecânicas (Fig.5 e 6). Não foi constatada a presença de papilas gustativas, porém, em contrapartida, foram encontrados botões gustativos (Fig.7), os quais estão diretamente ligados ao epitélio de revestimento.

Ainda descrevendo os cortes microscópicos do órgão, pode-se afirmar que a língua é uma estrutura muscular estriada revestida por estruturas que podem variam de acordo com cada região. Regularmente é revestida por epitélio pseudo estratificado cilíndrico, com células caliciformes não queratinizadas e com considerável presença de células mucosas. Internamente observa-se a presença de tecido conjuntivo frouxo na porção superficial e tecido conjuntivo denso não modelado na porção mais profunda. Identificou-se uma grande quantidade de vasos sanguíneos e linfáticos, porém, o mesmo não pode ser afirmado para estruturas nervosas, as quais foram encontradas em pouca quantidade. Abaixo dessas estruturas, encontra-se a camada muscular, a qual é constituída de feixes de músculo estriado esquelético.

No terço médio da cavidade orofaríngea e na porção rostral do corpo da língua encontra-se a glote (Glo) que liga a cavidade à traqueia (Tr). Esta tem início no corpo da língua e se estende até a raiz, sendo completamente recoberta pela mesma. Caudal a abertura da glote, observamos duas proeminências laringeais, as quais circundam a abertura da glote. A junção caudal da glote estende-se no terço médio e terço caudal da orofaringe. Essa junção promove a formação de uma proeminência mediana que encaixa-se

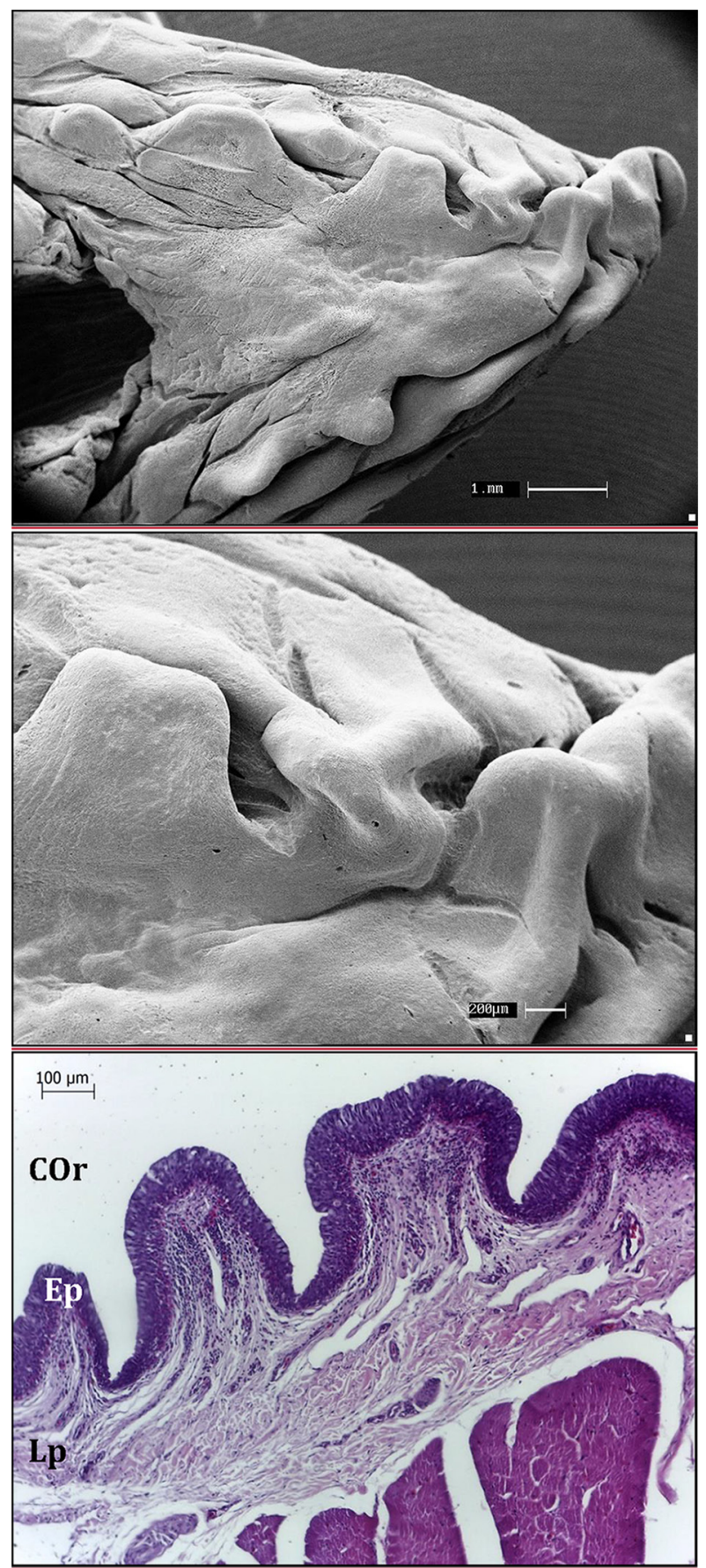

Fig.5. (A,B) Microscopia eletrônica de varredura da porção apical da língua da Tartaruga da Amazônia (Podocnemis expansa), onde é observado projeções do epitélio, sendo estas papilas linguais filiformes. (C) Fotomicrografia de corte transversal, da porção dorsal, do ápice da língua corado em HE evidenciando papilas linguais filiformes (seta).Cor, cavidade orofaríngea; Ep, epitélio de revestimento; Lp, lâmina própria.

perfeitamente nas protuberâncias do teto da orofaringe. Na porção lateral de ambos os lados é possível observar a articulação da mandíbula (Arm).

\section{DISCUSSÃO}

Segundo Ashley (1974) e Pinheiro et al. (2010) afirmam que as tartarugas possuem cabeças achatadas dorso-ven- 


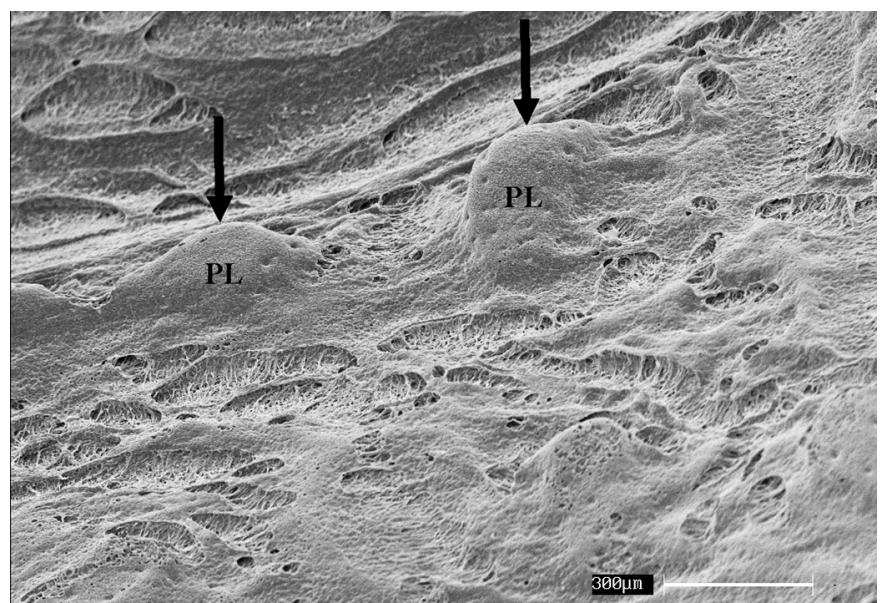

Fig.6. Microscopia eletrônica de varredura da superfície dorsal da língua da Tartaruga da Amazônia (Podocnemis expansa). Papilas linguais (PL) de forma abaulada, evidenciadas através das setas.

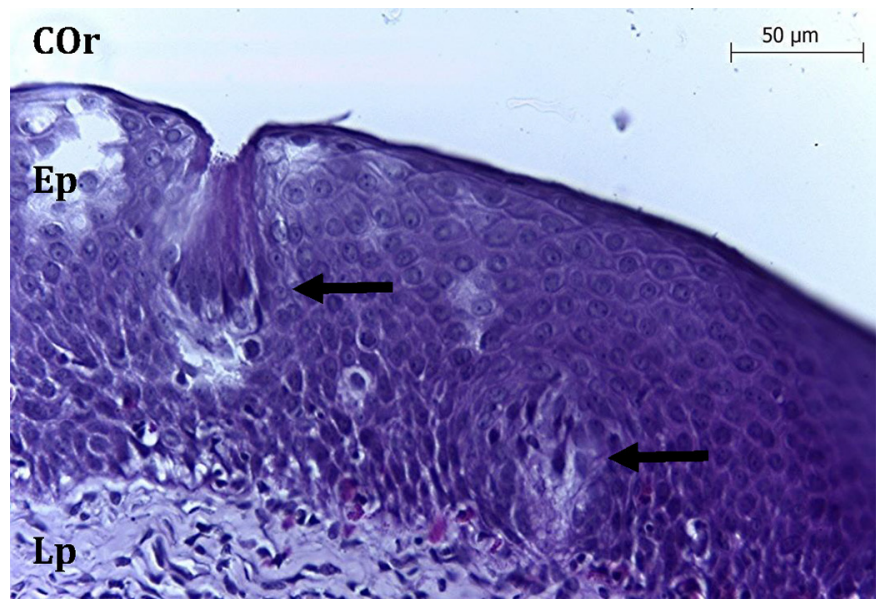

Fig.7. Fotomicrografia da superfície dorsal da porção apical da língua da Tartaruga da Amazônia (Podocnemis expansa), sendo possível observar os botões gustativos (setas) presentes no epitélio de revestimento. Cor, cavidade orofaríngea; Ep, epitélio de revestimento; Lp, lâmina própria.

tralmente, as quais representam uma estrutura triangular, semelhante a letra $\mathrm{V}$, tal que o formato da cavidade orofaríngea acompanha o formato da mesma, cujo comprimento é maior que a largura. Esses dados vão de encontro com os achados no presente estudo. Esses autores ainda afirmam que a cabeça da fêmea é maior a cabeça do macho, todavia no nosso estudo não foi observada diferença significativa entre os indivíduos estudados.

A família Cheloniidae é caracterizada por um crânio extenso que é coberto por uma ranfoteca bem desenvolvida. Esta é queratinosa, rígida e afiada, recobre estruturas ósseas como o osso maxilar, sendo responsável pela dilaceração dos alimentos, uma vez que os quelônios não possuem dentes. Ao analisar a ranfoteca maxilar e mandibular presente em Podocnemis expansa é possível afirmar que mesmo sendo estudadas espécies de ordens distintas, suas estruturas anatômicas são similares, pois desempenham as mesmas funções (Wyneken 2001, Witherington 2006, Natchev et al. 2011, Cubas et al. 2014).
As coanas de Podocnemis expansa se assemelham às de outras espécies, pois possuem formato elíptico e interligam a cavidade nasal e a cavidade orofaríngea, funcionando como comunicação para a passagem de ar (Pinheiro et al. 2010, Lintner et al. 2012). Os achados microscópicos da porção epitelial da porção dorsal da cavidade orofaríngea (a qual inclui a membrana que divide a abertura das coanas) se assemelham aos descritos por Aughey e Frye (2001), Dellman e Eurell (1998) e Samuelson (2007), uma vez que estes afirmam que a porção do epitélio que se estende ao longo da superfície da orofaringe é caracterizado como pavimentoso estratificado não queratinizado. De acordo com Pinheiro et al. (2010) as tubas auditivas se encontram caudalmente às coanas, nos antímeros direito e esquerdo no teto da cavidade orofaringea, o que vai de encontro aos achados de nosso estudo.

O bico da Podocnemis expansa é utilizado para a apreensão dos alimentos e para a defesa do animal. Associado a essa função temos o ângulo da mandíbula ("angle of jaw"), o qual é definido como uma estrutura óssea presente bilateralmente tanto no assoalho como no teto da cavidade orofaríngea que, juntamente com a musculatura potente presente na mandíbula, realiza a função de quebra do alimento. Essa descrição corrobora com a descrição dada a outros quelônios estudados (Ashley 1974, Luz \& Reis 1999, Mader 1996, Almeida 2011).

Quando observamos a musculatura presente na articulação da mandíbula de Podocnemis expansa com os estudos de Lintner et al. (2012) e Jones et al. (2012), P. expansa, semelhante às outras espécies de quelônios, não possui muitos grupos musculares na região mandibular. Em vez disso, há o músculo adutor da mandíbula, que possui diversos pontos de origem (cabeças), estas podem ser denominadas como: músculo adutor da mandíbula externo e músculo adutor da mandíbula interno.

Observa-se uma grande semelhança entre a língua de Podocnemis expansa com as espécies Manouria emys emys, Cuora galbinifrons e Mesoclemmys vanderhaegei, pois todas são alongadas, bem desenvolvidas, com seu ápice em formato rombo (triangular) e caudalmente encontra-se o frênulo proeminente, que permite uma grande aderência ao assoalho (Natchev et al. 2010, Pinheiro et al. 2010, Heiss et al. 2011). Natchev et al. (2011) acrescenta ainda que a retração do alimento pela língua começa a partir do momento que o alimento é liberado da ranfoteca durante a abertura da mandíbula, porém, antes de que o alimento flutue da cavidade oral. As características do epitélio da língua descritas por nós se assemelham às descritas por Aughey \& Frye (2001), Heiss (2010) e Samuelson (2007).

As papilas presentes na língua da espécie Manouria emys emys estudada por Heiss et al. (2011) macroscopicamente e em distribuição são muito semelhantes às analisadas nos nossos estudos. Ambas possuem formatos diversificados e estão espalhadas por toda superfície da estrutura. Já quando se compara Podocnemis expansa com a espécie Heosemys grandis nos estudos de Lintner et al. (2012), as duas possuem papilas que recobrem toda a língua, porém, na espécie Heosemys grandis há diferenciação entre as porções laterais e porção central, o que não observamos em P. expansa. 
Thewissen (2008) afirma que papilas gustativas podem ser encontradas distribuídas de forma difusa em diversas porções da língua de tartarugas, porém, não foram encontrados estudos que examinam o padrão dessas estruturas nesses animais. As papilas observadas nos nossos resultados mostraram-se como sendo mecânicas, pois não apresentam botões gustativos associados a essas. Entretanto, assume-se que a Tartaruga da Amazônia (Podocnemis expansa) possua paladar, uma vez que foram evidenciados botões gustativos no epitélio de revestimento da língua desses quelônios, fazendo com que essa diferença torne-se espécie-específica.

Microscopicamente, as papilas identificadas assemelham-se, em parte, com as papilas filiformes descritas por Junqueira e Carneiro (2003), pois, apesar de não possuírem o formato característico cônico e alongado, as mesmas não apresentam botões gustativos inseridos em seu epitélio.

Semelhante ao observado nos estudos de Sacchi et al. (2004) com a espécie Heosemys grandis, a glote é considerada a "porta de abertura" da laringe que caudalmente dá continuidade a traqueia, cuja parede é formada por anéis cartilaginosos completamente fechados.

De acordo com Almeida et al. (1986) e Armond (2008) as espécies vegetais são ingeridas pela Podocnemis expan$s a$ em suas formas inteiras, incluindo o sistema radicular (galhos). Nos estudos de Armond (2008) foram analisados aspectos alimentares da dieta de Podocnemis expansa em cativeiro, as quais havia presença de proteína animal; e em ambiente natural, tendo sido encontrado amostras predominantemente vegetais, reforçando assim a ideia de que $P$. expansa é uma espécie onívora oportunista, se alimentando de acordo com a disponibilidade do alimento. Malvasio et al. (2003) verificou que P. expansa se torna vegetariana com o decorrer da idade. Diante desses fatos, observamos a importância da cavidade orofaríngea associada aos hábitos alimentares desta espécie, uma vez que apesar de desprovida de dentes, Podocnemis expansa conta com a presença de rafontecas afiadas e bem desenvolvidas que, associadas a musculatura potente da mandíbula e a língua volumosa e bem distribuída no assoalho da orofaringe atuam na apreensão e deglutição do alimento, garantindo uma maior adaptação em diversos ambientes.

\section{REFERÊNCIAS}

Almeida C.G. 2011. Crescimento e digestibilidade de dietas com diferentes teores de fibra para a tartaruga-da-Amazônia, Podocnemis expansa. Tese de Doutorado em Aquicultura, Centro de Aquicultura, Universidade Estadual Paulista, Jaboticabal, SP. 111p.

Almeida S.S., Sá P.G.S. \& Garcia A. 1986. Vegetais utilizados como alimento por Podocnemis (Chelonia) na Região do Baixo Rio Xingu (Brasil-Pará). Bolm Mus. Pará Emílio Goeldi 2(2):199-211.

Armond F.N. 2008. Aspectos alimentares e do parasitismo em Podocnemis expansa de ambiente natural e criatório comercial no estado do Tocantins. Dissertação de Mestrado em Ciências do Ambiente, Fundação Universidade Federal do Tocantins, Palmas, TO. 164p.

Ashley L.M. 1974. Laboratory Anatomy of the Turtle. 10th ed. Wm. C. Brown Company Publishers, Dubuque. 100p.

Aughey E. \& Frye F.L. 2001. Comparative Veterinary Histology. Manson Publishing, London.
Brasil 1992. Portaria no 142 de 30 de dezembro de 1992, Ministério do Meio Ambiente, dos Recursos Hídricos e da Amazônia Legal. Disponível em <http://ibama.gov.br/category/49-_-_?download=1200\%3Ap-_ 142_92.p> Acesso em novembro de 2014.

Brasil 1996. Portaria no 70 de 23 de agosto de 1996, Ministério do Meio Ambiente, dos Recursos Hídricos e da Amazônia Legal. Disponível em <http://www.ibama.gov.br/fauna/legislacao/port_70_96.pdf> Acesso em outubro de 2014.

Cubas Z.S., Silva J.C.R. \& Catão-Dias J.L. 2014. Tratado de Animais Selvagens: medicina veterinária. $2^{\underline{a}}$ ed. Roca, São Paulo. 2512p.

Delmann H.D. \& Eurell J.A. 1998. Textbook of Veterinary Histology. Williams and Wilkins, Marylasnd.

Ernst C.H. \& Barbour R.W. 1989. Turtles of the World. Smithsonian Institution, Washington. 313p.

International Comittee on Veterinary Gross Anatomical Nomenclature 2005. Nomina Histologica Veterinaria. 2nd ed. Editorial Committee Hannover, Columbia. 166p.

Iverson J.B. 1992. A Revised Checklist with Distribution Maps of the Turtles of the World. Earlham College, Richmond. 363p.

Heiss E. 2010. Functionality and plasticity of turtle-feeding with special emphasis on oropharyngeal structures. Dissertation of Natural Sciences Doctoral Program of UniStG, Viena, Austria.

Heiss E., Natchev N., Schwaha T., Salaberg D., Lemell P., Beisser C. \& Weisgram J. 2011. Oropharyngeal morphology in the basal tortoise Manouria emys emys with comments on form and function of the testudine tongue. J. Morphol. 272:1217-1229.

Jones M.E.H., Werneburg I., Curtis N., Penrose R., O'Higgins P., Fagan M.J. \& Evans S.E. 2012. The Head and Neck Anatomy of Sea Turtles (Cryptodira: Chelonioidea) and Skull Shape in Testudines. PloS One 7(11):e47852. Doi:10.1371/journal.pone.0047852.

Lintner M., Weissenbacher A. \& Heiss E. 2012. The Oropharyngeal Morphology in the Semiaquatic Giant Asian Pond Turtle, Hepsemys grandis, and its Evolutionary Implications. PloS One 7(9):e46344. Doi: 10.1371/ journal.pone.0046344.

Luz V.L.F. 2005. Criação comercial de tartaruga e tracajá: manual técnico. Sebrae, Cuiabá. 72p.

Luz V.L.F. \& Reis I.J. 1999. Conservação e criação comercial de quelônios. Cenaqua, Goiânia. 34p.

Mader D.R. 1996. Reptile Medicine and Surgery. W.B. Saunders Company, Philadelphia. 512p.

Melo L.A.S., Izel A.C.U., Andrade P.C.M., Silva A.V. \& Hossaine-Lima M.G. 2003. Criação de Tartaruga da Amazônia (Podocnemis expansa). Embrapa. Disponível em <http://www.infoteca.cnptia.embrapa.br/handle/ doc/674013> Acesso em outubro de 2014.

Malvasio A., Souza A.M., Molina F.B. \& Sampaio F.A. 2003. Comportamento e preferência alimentar em Podocnemis expansa (Schweigger), $P$. unifilis (Troschel) e P. sextuberculata (Cornalia) em cativeiro (Testudines, Pelomedusidae). Revta Bras. Zool. 20(1):161-168.

Moura L.S.S. \& Rodrigues M.J.J. 2007. Análise bromatológica da carne de tartaruga-da-Amazônia, Podocnemis expansa (Schweigger, 1812) em habitat natural: subsídeos para otmizar a criação racional. Amazônia: Ciência e Desenvolvimento 2(4):1-16.

Natchev N., Lemell P., Heiss E., Beisser C. \& Weisgram J. 2010. Aquatic feeding in a terrestrial turtle: a funcional-morphological study of the feeding apparatus in the Indochinese box turtle Cuora galbinifrons (Testudines, Geoemydidae). Zoomorphology 119:111-119.

Natchev N., Heiss E., Singer K., Kummer S., Salaberger D. \& Weisgram J. 2011. Structure and function of feeding apparatus in the common musk turtle Sternotherus odoratus (Chelonia, Kinosternidae). Contrib. Zool. 80(2):143-156.

International Comitee on Veterinary Gross Anatomical Nomenclature 2012. Nomina Anatomica Veterinaria. 5th ed. Hannover. 165p.

Pinheiro J.N., Godoy I., Brito E.S., Strussmann C. \& Ferraz R.H.S. 2010. Macroscopia do aparelho digestório do cágado sul-americano Mesoclemmys vanderhaegei (Bour, 1973). Braz. J. Vet. Res. Anim. Sci. 47(6):429-438. 
Pritchard P.C.H. \& Trebbau P. 1984. The turtles of Venezuela. Society for the Study of Amphibians and Reptiles. Ithaca, New York. 415p.

Thewissen J.G.M. 2008. Sensory Evolution on the Threshold. California University.

Sacchi R., Galeotti P., Fasola M. \& Gerzeli G. 2004. Larynx morphology and sound production in three species of Testudinidae. J. Morphol. 261:175183.
Samuelson Don A. 2007. Tratado de Histologia Veterinária. Elsevier, Rio de Janeiro.

Witherington B.E. 2006. Sea Turtles: an extraordinary natural history of some uncommon turtles. Voyageur Press, London. 132p.

Wyneken J. 2001. The Anatomy of Sea Turtles. Disponível em <http:// www.sefsc.noaa.gov/turtles/TM_470_Wyneken.pdf> Acesso em novembro de 2014. 